\title{
Perfil e seguimento dos pacientes portadores de Mycobacterium sp. do Hospital das Clínicas da Universidade Federal de Minas Gerais*
}

\section{Profile and follow-up of patients with Mycobacterium sp. at the H ospital das Clínicas da U niversidade Federal de Minas Gerais}

\author{
Giselle Carvalho Froes ${ }^{1}$, Rosane Luiza Coutinho ${ }^{1}$, Marcelo Nardy de Ávila ${ }^{1}$, \\ LEANDRA ROCHA CANÇADO ${ }^{1}$, SILVANa SPÍNDOLA DE MIRANDA ${ }^{2}$
}

\begin{abstract}
Introdução: Em levantamentos feitos no Laboratório de Micobactérias do H ospital das Clínicas da U niversidade Federal de Minas Gerais, em anos anteriores, constatou-se que cerca de $42 \%$ dos pacientes portadores de micobacterioses não haviam iniciado o tratamento por desconhecerem o diagnóstico.
\end{abstract}

O bjetivo: Avaliar o perfil dos pacientes portadores de micobacterioses do H ospital das Clínicas da U niversidade Federal de Minas Gerais. Descrever o seguimento desses pacientes e sua inserção no sistema de saúde, comparando com a proposta do Programa Nacional de Controle da Tuberculose.

Método: A partir dos registros do Laboratório Central do Hospital das Clínicas da Universidade Federal de Minas Gerais de janeiro a dezembro de 2002, os autores selecionaram os pacientes com diagnóstico bacteriológico de micobacterioses e efetuaram a busca ativa e o seguimento desses pacientes.

Resultados: De um total de 66 pacientes, 62 (94\%) tinham cepas do Mycobacterium tuberculosis e quatro $(6 \%)$, micobactéria não tuberculosa; quatro desses 62 pacientes $(6 \%)$ foram transferidos para outras instituições. A pós a busca ativa, de um total de 58 pacientes, 37 (63\%) estavam curados, 11 (19\%) faleceram, sendo que destes, sete $(64 \%)$ eram positivos para o vírus da imunodeficiência humana (HIV), um (2\%) recusou tratamento e nove (16\%) não foram encontrados.

Conclusão: Não foi a falta de diagnóstico o que impossibilitou o paciente de receber seu tratamento e, sim, uma estrutura inadequada, com baixa conscientização de todos os envolvidos, demonstrando a desorganização no controle da tuberculose em nível hospitalar. A cura dos pacientes foi aquém da exigida pelo Ministério da Saúde, devido à grande quantidade de soropositivos para o HIV. Os óbitos foram relacionados ao HIV e ao desconhecimento da doença. É fundamental que os profissionais que atuam em nível laboratorial realizem pesquisas operacionais em tuberculose em conjunto com profissionais da área clínica, pois somente dessa forma serão identificadas respostas aos problemas da prática clínico-laboratorial em nosso meio. (J Pneumol 2003;29(6):365-70)

Descritores - Tuberculose/ diagnóstico. Micobacteriose/ complicações.
Background: Surveys of patients diagnosed with mycobacteriosis, taken at the Laboratory of Mycobacteriology of the Hospital das C línicas da U niversidade Federal de Minas Gerais, have shown that lack of information concerning diagnosis prevented $42 \%$ from initiating treatment.

O bjective: To evaluate the profile of patients with mycobacterial infection attending the Hospital das Clínicas da U niversidade Federal de Minas Gerais. To describe the follow up of those patients and compare it to the guidelines made by the National Program for the Control of Tuberculosis.

Methods: The files of patients diagnosed with mycobacterium infection during 2002 were selected from the archives of the Laboratory of Mycobacteriology of the H ospital das Clínicas da Universidade Federal de Minas Gerais. An active search for these patients was carried out and follow-up exams were then performed.

Results: Of the 66 patients selected, 62 (94\%) were positive for Mycobacterium tuberculosis and $4(6 \%)$ had nontuberculous mycobacteriosis. A nother $4(6 \%)$ had been transferred to other institutions. Of the remaining 58, $37(63 \%)$ had been cured, $1(2 \%)$ had refused treatment, 9 (16\%) were not found and 11 (19\%) had died. Of the 11 deaths, 7 (64\%) had tested positive for HIV.

Conclusion: Patients failed to receive treatment not due to lack of diagnosis, but to inadequate structure, low awareness levels of all parties, and lack of tuberculosis control organization at the hospital level. Due to the high number of HIV-positive patients, the number of patients cured was lower than that required by the $\mathrm{Na}$ tional $\mathrm{H}$ ealth Ministry. Deaths were attributed to HIV infection and lack of knowledge about the disease. In order to identify and address the problems associated with clinical laboratory practice, laboratory professionals must work in concert with their clinical counterparts when carrying out operational research on tuberculosis.

Key words - Tuberculosis/ diagnosis. Mycobacterium infections/ complications
* Trabalho realizado no H ospital das Clínicas da U niversidade Federal
de Minas Gerais.

1. Graduando de Medicina Iniciação Científica.

2. Coordenadora de Pesquisa em Micobacterioses.
Endereço para correspondência - Silvana Spíndola de Miranda, Av. Alfredo Balena, 120 - Santa Efigênia. Departamento de Clínica Médica/ Pneumologia, 4a and. - 30103-100. Tel.: (31) 3248-9599; E-mail: spindola@medicina.ufmg.br

Recebido para publicação em 16/6/03. Aprovado, após revisão, em 25/8/03. 


\section{Veja cometários no Editorial, na página 337}

\section{INTRODUÇÃO}

A tuberculose (TB) é hoje a primeira causa de morte no mundo entre mulheres de 15 a 44 anos e a segunda entre os homens da mesma faixa etária. Em países em desenvolvimento, é responsável por maior número de mortes que todas as outras doenças infecto-contagiosas juntas, a despeito de ser uma doença cujo tratamento oferece $97 \%$ de eficácia.(1)

Estima-se que 40 a 50 mil óbitos anuais são causados pela tuberculose na América. A situação é grave, principalmente porque se estima que $20 \%$ a $30 \%$ dos tuberculosos com alto risco de morrer não são notificados nem tratados. No Brasil, durante o ano de 2001, notificou-se um total de 82.866 casos de tuberculose, o que correspondeu a um coeficiente de incidência de 42,28 por 100.000 habitantes, sendo que $24,40 / 100.000$ constituem os casos pulmonares positivos ao exame de escarro, $11,75 / 100.000$ os casos pulmonares sem confirmação bacteriológica e 5,88/100.000 os casos de tuberculose extrapulmonar. ${ }^{(2)} \mathrm{A}$ razão de acometimento da tuberculose dos homens em relação às mulheres, de 1998 a 2000, foi de 1,8:1.(3) A taxa de mortalidade no Brasil para 0 ano de 2001 foi de $3,07 / 100.000$ habitantes, correspondendo a 5.294 óbitos. Entretanto, a taxa estimada é bem superior, de $11,0 / 100.000$ habitantes. ${ }^{(1,2)}$ A pesar de ter havido redução significativa da taxa de óbitos no Brasil desde 1977, a situação ainda é preocupante. Em Minas Gerais, a incidência da doença no ano de 2001 foi de 34,1/100.000 habitantes e, em Belo Horizonte, de $61,71 / 100.000$ habitantes. ${ }^{(4)}$

Segundo a Organização Mundial de Saúde (OMS), em 1999 foram estimados 637 mil casos de tuberculose associados ao vírus da imunodeficiência humana (HIV). $\mathrm{Na}$ A mérica Latina, África e em algumas populações de países desenvolvidos, $30 \%$ a $60 \%$ dos infectados pelo HIV estão co-infectados pelo bacilo da tuberculose. (1) No Brasil, entre 1980 e junho de 2000, ocorreram 190.523 casos de síndrome da imunodeficiência adquirida (AIDS). Estima-se que, destes, 20\% a 40\% desenvolveram tuberculose. (1) Em Belo Horizonte, $9 \%$ dos pacientes notificados com tuberculose são portadores do HIV.(5)

O Ministério da Saúde propõe procurar os casos de TB, diagnosticar principalmente os bacilíferos, tratar adequadamente os doentes, prevenir a doença e estabelecer medidas de biossegurança. A meta é identificar $90 \%$ dos casos e curar $85 \%$ dos pacientes até 2005. ${ }^{(6)}$

No Hospital das Clínicas da U niversidade Federal de Minas Gerais (HC/ UFMG), diagnosticamos por baciloscopia e/ ou cultura cerca de 60 casos de tuberculose anual-
Siglas e abreviaturas utilizadas neste trabal ho

AIDS - Síndrome da imunodeficiência adquirida

HC/ UFMG - H ospital das Clínicas da Universidade Federal de Minas Gerais

HIV - Vírus da imunodeficiência humana

MNT - Micobactéria não tuberculosa

Mtb - Mycobacterium tuberculosis

OMS - Organização Mundial de Saúde

PNCT - Programa Nacional de Controle da Tuberculose

TB - Tuberculose

mente. Esses pacientes são provenientes das enfermarias, pronto-atendimento (PA) do HC/UFMG, dos ambulatórios (HC/ UFMG) e do Centro de Referência O restes Diniz/ HIV/ AIDS (CTR/ HIV/ AIDS).

Em levantamentos feitos no Laboratório de Micobactérias do Hospital das Clínicas da UFMG em anos anteriores, observamos que cerca de $42 \%$ dos pacientes portadores de micobacterioses não tinham iniciado seus tratamentos, pois desconheciam o diagnóstico, mostrando, assim, a desorganização no controle da tuberculose em nível hospitalar. ${ }^{(7)}$

Tendo observado esse dado preocupante, iniciamos em nosso complexo hospitalar universitário a reestruturação dos setores que recebem pacientes sintomáticos respiratórios. A valiamos o perfil dos pacientes com diagnóstico bacteriológico de micobacterioses do HC/ UFMG durante 0 ano de 2002, com especial interesse no seguimento desses pacientes, e na sua busca e inserção no sistema de saúde, de acordo com as propostas do Programa Nacional de Controle da Tuberculose (PNCT). ${ }^{(8)}$

\section{MÉTOdo}

Selecionamos os pacientes com amostras clínicas positivas (baciloscopia e/ ou cultura) através do registro no Laboratório de Micobactérias no período de janeiro a dezembro de 2002 . O btivemos um total de 2.279 amostras, correspondendo a aproximadamente 700 indivíduos. Foram incluídos os diagnósticos de Mycobacterium tuberculosis ( $\mathrm{M} \mathrm{tb}$ ) e de micobactéria não tuberculosa (MNT). A baciloscopia foi feita pelas técnicas de Ziehl-Neelsen e fluorescência, a cultura em meio de Lowenstein-J ensen. 0 teste de identificação foi feito através de testes bioquímicos e o teste de sensibilidade, pelo método de proporção. A mbos foram realizados em todas as amostras positivas em cultura. ${ }^{(9,10)}$

Em 66 pacientes as amostras clínicas foram positivas pela baciloscopia e/ ou cultura para micobactérias. Em quatro (6\%) amostras foi identificada MNT; $19,5 \%$ do diag- 
nóstico bacteriológico dos pacientes com M tb foram obtidos somente pela cultura, como demonstrado na Tabela 1.

A partir dos registros do laboratório, iniciamos a busca dos pacientes, como demonstrado no fluxograma da Figura 1.

A valiamos o perfil dos 66 pacientes com diagnóstico bacteriológico de micobacterioses quanto às seguintes variáveis: gênero, idade, procedência, passado de tuberculose, clínica sugestiva de tuberculose, perfil radiológiCO, status imunológico para o HIV (ELISA e Western Blot) e seguimento (cura, falência de tratamento, abandono, transferência, óbito e pacientes não encontrados). Consi-

TABELA 1

Diagnóstico bacteriológico dos 62 pacientes com Mtb

\begin{tabular}{lccrc}
\hline & $\begin{array}{c}\text { Baciloscopia } \\
\text { positiva }\end{array}$ & $\begin{array}{c}\text { Baciloscopia } \\
\text { negativa }\end{array}$ & Total \\
\hline Cultura positiva & $49(79 \%)$ & $12(19,5 \%)$ & 61 & $(98,5 \%)$ \\
Cultura negativa & $1(1,5 \%)$ & $0(0 \%)$ & 1 & $(1,5 \%)$ \\
Total & $50(60,5 \%)$ & $12(19,5 \%)$ & $62(100 \%)$ \\
\hline
\end{tabular}

deramos os pacientes para os quais a sorologia anti-H IV não foi solicitada e os que estavam aguardando o resultado como tendo sorologia desconhecida.

Dos 66 pacientes, 27 (41\%) eram provenientes do H ospital das Clínicas (21\% do pronto-atendimento e $20 \%$ das enfermarias); 37 (56\%), dos ambulatórios; e dois (3\%), de origem desconhecida.

Os prontuários foram revisados após seis meses do início do tratamento para concluir o caso.

Todos os pacientes encontrados foram convidados a responder a um questionário, após consentimento, em que foram abordadas questões referentes aos dados sociais, clínicos e epidemiológicos (Comitê de Ética da UFMG, parecer no 162/01).

\section{Resultados}

Dentre os 62 pacientes com M tb, 35 (56,5\%) eram do sexo masculino e $27(43,5 \%)$ do feminino $(1,3: 1)$. Dos quatro pacientes com MNT, três eram do sexo masculino e um do feminino.

Foi possível encontrar os prontuários de 58 (88\%) dos 66 pacientes. Destes, 54 tinham M tb. A idade variou de

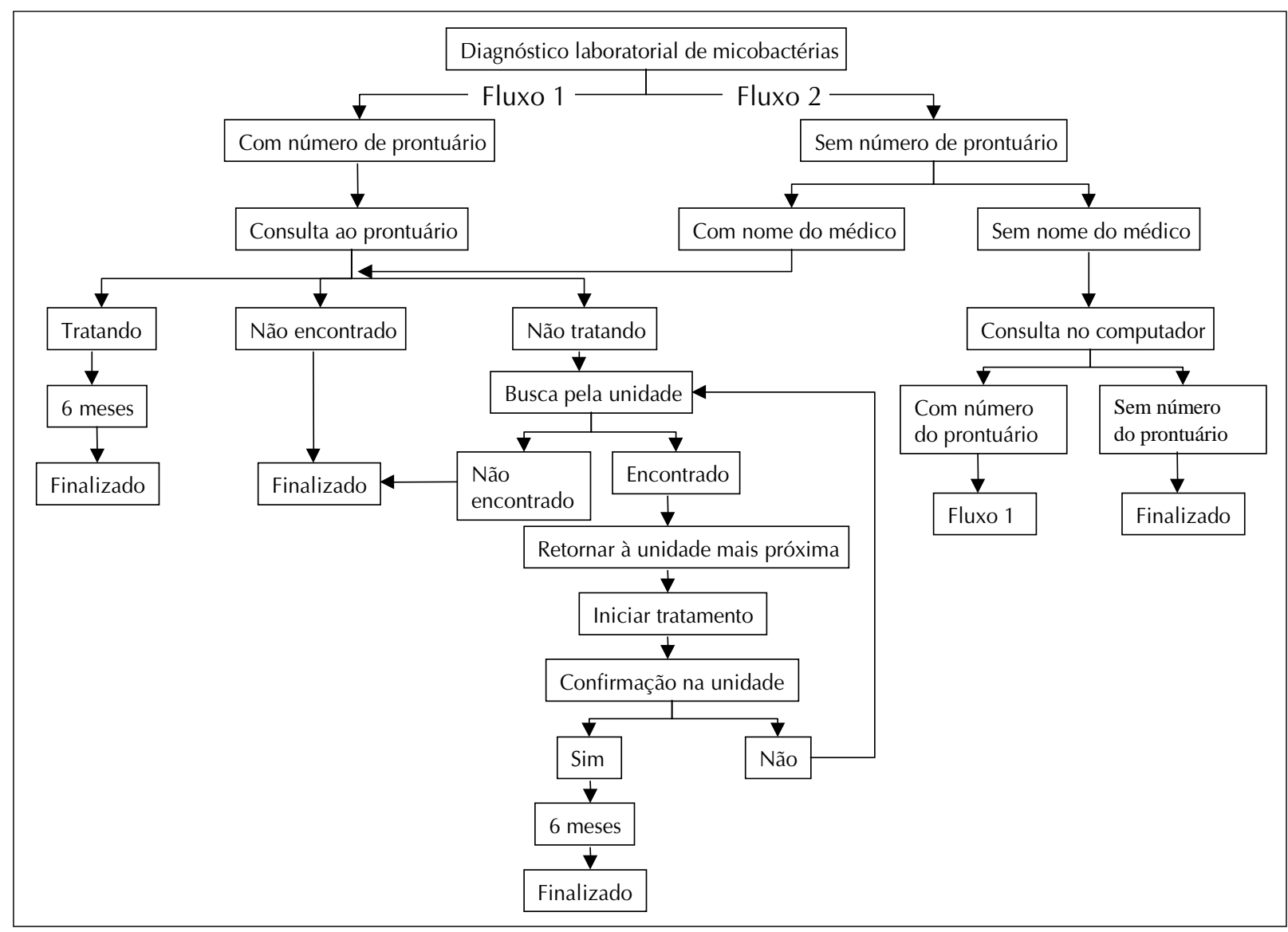

Figura 1 - Fluxo de busca de pacientes 
19 a 84 anos, com mediana de 35 anos, estando a maioria dos acometidos entre 25 e 57 anos. Q uanto à procedência, dos 54 pacientes com Mtb, 32 (59\%) eram de Belo Horizonte (MG); nove (17\%), da região metropolitana de Belo Horizonte; 12 (22\%), de outras cidades de Minas Gerais; e um (2\%), de outro Estado.

Dos 54 pacientes com M tb que tiveram seus prontuários revistos, $53(98 \%)$ apresentavam clínica sugestiva de tuberculose e um (2\%) não possuía essa informação em seu prontuário.

Um paciente tinha TB renal. Dos 53 pacientes com diagnóstico de TB pulmonar, a radiografia de tórax foi compatível em 46 (87\%) deles. Não foram encontradas as radiografias de sete $(13 \%)$ desses pacientes.

Os pacientes bacilíferos iniciaram o tratamento em 11,4 dias, em média.

Foram entrevistados 29 (54\%) dos 54 pacientes com $M$ tb, sendo que apenas quatro ( $14 \%)$ destes relataram passado de tuberculose.

Eram soropositivos para o HIV 25 (46\%) dos 54 pacientes, sendo que, destes, 17 (68\%) eram do sexo masculino e oito $(32 \%)$ do feminino. Do total de 54 pacientes, nove $(17 \%)$ eram soronegativos e 20 (37\%) tinham sorologia desconhecida (três com sorologia em andamento e 17, cuja sorologia não foi solicitada). Dos 11 pacientes portadores de Mtb que faleceram, sete eram HIV positivos.

O s oito (15\%) pacientes que não tinham conhecimento do diagnóstico de tuberculose foram procurados pela unidade de saúde, o que resultou nos seguintes dados: um teve alta por cura, um recusou o tratamento, três não foram encontrados e três faleceram.

A té o momento em que os casos foram acompanhados, não foi observada falência de tratamento ou abandono.

Dos 62 pacientes com diagnóstico de $M$ tb, quatro foram transferidos. 0 seguimento dos demais 58 pacientes está demonstrado na Figura 2.

Os quatro pacientes em retratamento eram HIV negativos e todos curaram-se. Excluindo-se esses pacientes, a taxa de cura total seria de $61 \%(33 / 54)$.

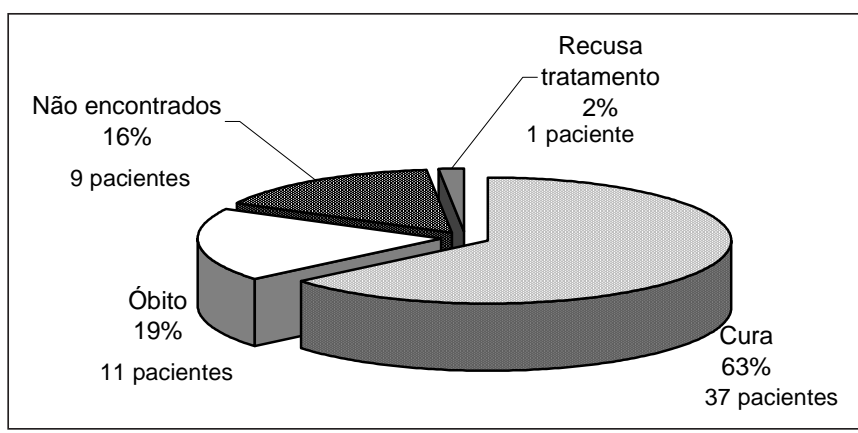

Figura 2 - Seguimento dos 58 pacientes com M icobacterium tuberculosis
$\mathrm{Na}$ Tabela 2 apresentamos as taxas de cura dos pacientes que tiveram seus prontuários encontrados.

Em quatro $(6 \%)$ dos 66 casos as micobactérias eram não tuberculosas, sendo que um desses pacientes, que era soropositivo para o HIV, faleceu.

\section{DISCUSSÃO}

Neste estudo, partimos do diagnóstico laboratorial de Mtb, como demonstrado na Tabela 1, porém, cabem algumas considerações. Em nosso laboratório, 80,5\% dos pacientes tiveram o diagnóstico de TB apenas através da baciloscopia. A cultura elevou a taxa de diagnóstico em $19,4 \%$, o que demonstra a necessidade de sua realização em nosso hospital. U ma amostra foi insuficiente para realizar a cultura, porém, consideramos que o paciente tinha o diagnóstico de tuberculose, pois evoluiu para cura após tratamento com o esquema I. ${ }^{\left({ }^{8}\right)}$ É também necessária a implantação de novas técnicas efetivas e rápidas para 0 diagnóstico da tuberculose. ${ }^{(11,12)}$

Recebemos no HC/ UFM principalmente pacientes procedentes de Belo H orizonte e região metropolitana. U ma grande percentagem de pacientes era proveniente do pronto-atendimento $(21 \%)$ e enfermarias $(20 \%)$. Portanto, a porta de entrada desses pacientes foi a unidade de pronto-atendimento. Isso reflete as deficiências no atendimento primário, eleva a casuística de morbimortalidade e expõe os profissionais de saúde ao risco de infecção pelo $\mathrm{M}$ tb, já que a maioria dos hospitais não possui estratégias de biossegurança. ${ }^{(11)}$

Dentre os pacientes acometidos pela tuberculose, não observamos diferença significativa na proporção entre homens e mulheres $(1,3: 1)$. Esse fato poderia ser explicado pela nossa pequena casuística e/ ou pela disputa no mercado de trabalho, que culmina com a saída das muIheres de sua residência e maior exposição aos riscos, biológicos ou não, da sociedade contemporânea.

A tuberculose acometeu principalmente indivíduos da faixa etária produtiva entre 25 e 57 anos, o que reflete a contribuição da doença para o comprometimento do quadro econômico do país. $(1,11)$

Em nossa casuística, $46 \%$ dos pacientes com tuberculose eram portadores do HIV, sendo a freqüência desse vírus maior no sexo masculino, em concordância com os

TABELA 2

Alta por cura dos pacientes com Mtb

\begin{tabular}{lccc}
\hline & Total de pacientes & \multicolumn{2}{c}{ Cura } \\
\hline HIV + & 25 & $14 / 25$ & $(56 \%)$ \\
HIV - & 9 & $9 / 9$ & $(100 \%)$ \\
Sorologia desconhecida & 20 & $14 / 20$ & $(70 \%)$ \\
Total & 54 & $37 / 54$ & $(69 \%)$ \\
\hline
\end{tabular}


dados da literatura. ${ }^{(11-13)}$ O bservamos que a taxa de positividade para o HIV encontrada em pacientes com tuberculose atendidos no hospital foi muito superior àquela referida nos atendidos nas unidades primárias de saúde. A lém disso, a maioria dos óbitos ocorreu em pacientes HIV positivos. Devemos ressaltar, entretanto, que nossa casuística é influenciada pelo recebimento de pacientes do CTR/ HIV/ AIDS.

O desconhecimento do status imunológico de $37 \%$ dos pacientes com Mtb foi devido, na maioria dos casos, à não solicitação do exame. Isso pode refletir a falta de informação ou baixa conscientização dos profissionais de saúde e a ausência de uma rotina adequada em hospitais gerais.

Neste trabalho observamos que a alta por cura está aquém da meta esperada pelo Ministério da Saúde, que seria de $85 \%$ de cura dos casos em que se utiliza o esquema I. ${ }^{\left({ }^{8}\right)}$ Esse resultado poderia ser justificado pela grande percentagem de pacientes HIV positivos e pelo desconhecimento da sorologia pela sua maioria. Se excluíssemos os pacientes com retratamento, a taxa de cura seria menor $(61 \%)$, já que, destes, todos se curaram.

Nos prontuários revistos, a clínica e as radiografias de tórax dos pacientes eram compatíveis com tuberculose, mesmo nos pacientes soropositivos para o H IV. Isso sugere que, com o uso do esquema anti-retroviral de alta potência introduzido no Brasil no final de 1996, eles passaram a se comportar como pacientes imunocompetentes, diferentemente do que foi observado em outro hospital universitário no Rio de J aneiro (RJ).(14)

Um dos maiores problemas na busca dos prontuários foi a falta de dados nos pedidos dos exames, tais como o número de prontuário, o nome completo do paciente, ou o nome do médico. A procura das fichas provenientes do PA constituiu outro problema, pois os pacientes que lá permanecem por menos de $24 \mathrm{~h}$ não têm prontuários e suas fichas não são arquivadas de maneira organizada, o que tornou impossível encontrá-las. 0 certo é que existe uma desorganização evidente, que está sendo revista e melhorada.

O preenchimento inadequado dos prontuários é histórico, o que muitas vezes inviabiliza vários estudos. Em vista disso, temos uma proposta de criar fichas com facilidade de preenchimento para amenizar o problema. Também enfrentamos algumas recusas para o preenchimento do questionário, o que é normal dentro de qualquer estudo, embora os pacientes que concederam a entrevista tivessem tido o mesmo atendimento e atenção por parte dos pesquisadores quanto aqueles que se recusaram a concedê-la.

Os pacientes que não tinham conhecimento do diagnóstico $(15 \%)$ foram exaustivamente procurados por nós e pela unidade de saúde, sendo que alguns foram encon- trados tardiamente e outros já haviam falecido sem que tivessem iniciado o tratamento. U m grande esforço deve ser feito na organização do sistema para facilitar e meIhorar o encontro desses pacientes, que também devem ser orientados a retornar para buscar os resultados da cultura quando de suas baciloscopias negativas.

U m paciente recusou o tratamento apesar dos esforços em convencê-lo a iniciá-lo, porém, a equipe de saúde continuou trabalhando no caso.

Poucos casos foram diagnosticados com MNT. Três tinham suspeita de colonização ou contaminação (um com síndrome de Sjögren, um com lúpus eritematoso sistêmico e um HIV positivo) e todos evoluíram bem sem tratamento. O paciente HIV positivo teve doença disseminada por MNT (isolada de sítio estéril), falecendo em poucas semanas.

A média de 11,4 dias entre o diagnóstico e o início do tratamento é elevada. Muito tem que ser feito para que pacientes com diagnóstico confirmado de tuberculose sejam tratados o mais rápido possível, impedindo a cadeia de transmissão do bacilo. Entretanto, recursos humanos e financeiros para que possamos efetivamente controlar a doença muitas vezes não chegam aos setores interessados.

A criação do fluxograma permitiu melhor avaliação do perfil e do seguimento dos pacientes com Mycobacterium sp. do HC/UFMG. Sendo assim, ele pode vir a ser útil também para outros serviços. Podemos acrescentar a esse fluxograma a busca no Sistema de Informação da Secretaria Estadual de Saúde, o que permitiria recuperar os casos não encontrados, mas notificados em outras unidades.

Enquanto não for estabelecida pelos formuladores de políticas públicas do Ministério da Saúde a implantação de programas de controle de tuberculose em nível hospitalar, com características de atuações em multidisciplinaridade, incluindo questões de diagnóstico, tratamento e biossegurança, erros vão-se repetir. Este estudo demonstrou que não foi a falta de diagnóstico que impossibilitou o paciente de receber seu tratamento, mas, sim, uma estrutura inadequada, com baixa conscientização de todos os envolvidos. Nenhum outro estudo que descreva o que acontece após o diagnóstico laboratorial foi encontrado após levantamento da literatura nacional. É fundamental que os profissionais que atuam em nível laboratorial realizem pesquisas operacionais em tuberculose juntamente com profissionais da área clínica, pois somente dessa forma serão encontradas respostas para os problemas da prática clínico-laboratorial.

\section{Agradecimentos}

Agradecemos aos funcionários do Laboratório Central do H ospital das Clínicas da UFMG, setor de Micobactérias, aos pro- 
fissionais e, principalmente, aos pacientes que contribuíram de forma direta ou indireta para a realização deste trabalho. Também, em especial, ao Professor Afrânio Lineu Kritski, da Universidade Federal do Rio de J aneiro, pela revisão do texto.

\section{REFERÊNCIAS}

1. Ministério da Saúde. Fundação Nacional de Saúde. Centro de Referência Prof. Hélio Fraga/ Sociedade B rasileira de Pneumologia e Tisiologia. Controle da tuberculose - U ma proposta de integração ensinoserviço. 5a ed. Rio de J aneiro; 2002.

2. Ministério da Saúde. Disponível em: http:// dtr2001.saude.gov.br/sps/ areastecnicas.

3. Ministério da Saúde. Secretaria de Políticas de Saúde. Situação da tuberculose no Brasil. Brasília, 2002.

4. DATASUS. Disponível em: http:// tabnet. datasus.gov.br.

5. Dados do Sistema de Informação da Secretaria Estadual de Saúde de Minas Gerais. Belo Horizonte, 2001.

6. Ministério da Saúde. Fundação Nacional de Saúde. Tuberculose - Guia de vigilância epidemiológica. Brasília, 2002.

7. A njos Moreira J , Froes GC, Spindola de Miranda S. Cases of tuberculosis diagnosed at Federal University Hospital, Belo Horizonte, MG,
Brazil. In: 33rd World Conference on Lung $\mathrm{H}$ ealth of the International Union Against Tuberculosis and Lung Disease (IUATLD). Montreal, Canada, 6-10 Oct. 2002. Int J Tuberc Lung Dis 2002;6(10 Suppl 1): S1-210.

8. Ministério da Saúde. Fundação Nacional de Saúde. Plano Nacional de Controle da Tuberculose - Normas técnicas, estrutura e operacionalização. B rasília; 2000.

9. Ministério da Saúde. Fundação Nacional de Saúde. Centro de Referência Prof. Hélio Fraga. Manual de bacteriologia da tuberculose. 2a ed. Rio de J aneiro, 1994.

10. Canetti G, Rist N, Grosset J. Mesure da la sensibilité du bacille de la tuberculeux aux antibacillares par la méthode des proportions. Rev Tuberc Pneumol 1963;27:217.

11. Kritski $A L$, Conde $M B$, Souza GRM. Tuberculose: do ambulatório à enfermaria. 2a ed. São Paulo: A theneu, 2000.

12. Kritski AL, Lapa e Silva JR, Conde MB. Tuberculosis and HIV: renewed challenge. Mem Inst O swaldo Cruz 1998;93:417-21.

13. Muzy de Souza GR, Gonçalves M, Carvalho ACC. Controle de infecção hospitalar por tuberculose. Pulmão RJ 1997;6:220-7.

14. Mello FCQ, Lacerda APM, Gounder C, Salles CLG, Madeira FB, Gomes SA, Kritski A. The impact of institution of HAART in the coinfection tuberculosis and HIV in a reference hospital for AIDS in Rio de J aneiro, Brazil. Am J Respir Crit Care Med 2001;163:A496. 